\title{
Neurotransmitter Release Can Be Stabilized by a Mechanism That Prevents Voltage Changes Near the End of Action Potentials from Affecting Calcium Currents
}

\author{
Stephen G. Clarke, ${ }^{1,2}{ }^{\circledR}$ Matthew S. Scarnati, ${ }^{1}$ and ${ }^{\circledR}$ Kenneth G. Paradiso ${ }^{1}$ \\ ${ }^{1}$ Department of Cell Biology and Neuroscience and ${ }^{2}$ Graduate Program in Biomedical Engineering, Rutgers University, Piscataway, New Jersey 08854
}

At chemical synapses, presynaptic action potentials (APs) activate voltage-gated calcium channels, allowing calcium to enter and trigger neurotransmitter release. The duration, peak amplitude, and shape of the AP falling phase alter calcium entry, which can affect neurotransmitter release significantly. In many neurons, APs do not immediately return to the resting potential, but instead exhibit a period of depolarization or hyperpolarization referred to as an afterpotential. We hypothesized that presynaptic afterpotentials should alter neurotransmitter release by affecting the electrical driving force for calcium entry and calcium channel gating. In support of this, presynaptic calcium entry is affected by afterpotentials after standard instant voltage jumps. Here, we used the mouse calyx of Held synapse, which allows simultaneous presynaptic and postsynaptic patch-clamp recording, to show that the postsynaptic response is affected significantly by presynaptic afterpotentials after voltage jumps. We therefore tested the effects of presynaptic afterpotentials using simultaneous presynaptic and postsynaptic recordings and AP waveforms or real APs. Surprisingly, presynaptic afterpotentials after AP stimuli did not alter calcium channel responses or neurotransmitter release appreciably. We show that the AP repolarization time course causes afterpotential-induced changes in calcium driving force and changes in calcium channel gating to effectively cancel each other out. This mechanism, in which electrical driving force is balanced by channel gating, prevents changes in calcium influx from occurring at the end of the AP and therefore acts to stabilize synaptic transmission. In addition, this mechanism can act to stabilize neurotransmitter release when the presynaptic resting potential changes.

Key words: action potential; afterpotential; calcium current; neurotransmission; presynaptic; synapse

Significance Statement

The shape of presynaptic action potentials (APs), particularly the falling phase, affects calcium entry and small changes in calcium influx can produce large changes in postsynaptic responses. We hypothesized that afterpotentials, which often follow APs, affect calcium entry and neurotransmitter release. We tested this in calyx of Held nerve terminals, which allow simultaneous recording of presynaptic calcium currents and postsynaptic responses. Surprisingly, presynaptic afterpotentials did not alter calcium current or neurotransmitter release. We show that the AP falling phase causes afterpotential-induced changes in electrical driving force and calcium channel gating to cancel each other out. This mechanism regulates calcium entry at the end of APs and therefore stabilizes synaptic transmission. This also stabilizes responses when the presynaptic resting potential changes.

\section{Introduction}

Changes in the shape, duration, and amplitude of the action potential (AP) affect calcium entry by affecting calcium channel

Received Jan. 7, 2016; revised Aug. 29, 2016; accepted Sept. 14, 2016

Author contributions: K.G.P. designed research;S.G.C., M.S.S., and K.G.P. performed research; S.G.C., M.S.S., and K.G.P. analyzed data; K.G.P. wrote the paper.

This work was supported by the National Institutes of Health (Grant R00 NS051401-42 to K.G.P. We thank Dr. L. Milescu and Dr. D. Robinson for critical reading of this manuscript and helpful comments and Dr. M. Plummer for helpful discussions on this work.

The authors declare no competing financial interests.

Correspondence should be addressed to Kenneth G. Paradiso, Department of Cell Biology and Neuroscience, Rutgers University, 604 Allison Rd., Nelson D423, Piscataway, NJ 08854. E-mail: kenparadisolab@gmail.com. activation, channel gating, and the electrical driving force for calcium entry (Pattillo et al., 1999; Geiger and Jonas, 2000; Bischofberger et al., 2002; Debanne, 2004; Wu et al., 2004; Bean, 2007; Kole et al., 2007; Hoppa et al., 2014). In addition, relatively small changes in presynaptic calcium influx can produce large changes in the postsynaptic response (Bollmann et al., 2000; Schneggenburger and Neher, 2000; Fernández-Chacón R et al., 2001; Eggermann et al., 2012). Therefore, the shape, duration, and am-

DOI:10.1523/JNEUROSCI.0066-16.2016

Copyright $\odot 2016$ the authors $\quad 0270-6474 / 16 / 3611559-14 \$ 15.00 / 0$ 
plitude of the presynaptic AP are critical components in determining the strength of synaptic transmission.

In many neurons, the repolarization phase of the AP does not return directly to the resting potential. Instead, afterpotentials (Gasser and Erlanger, 1930) often occur at the end of the AP repolarization such that the membrane potential remains transiently at a level slightly above or below the resting potential (Bean, 2007; Debanne et al., 2011). Afterpotentials can facilitate (Paradiso and Wu, 2009; Lewis and Raman, 2014) or suppress AP firing (Kim et al., 2007; Paradiso and Wu, 2009). A brief afterhyperpolarization (AHP) can reduce the time required for voltagegated ion channels to recover from inactivation (Rudy and McBain, 2001; Kopp-Scheinpflug et al., 2011), which can allow higher firing frequencies. In addition, afterpotentials should affect calcium channel currents because the majority of calcium entry occurs during the repolarization phase of the $\mathrm{AP}$ (Borst and Sakmann, 1998, 1999; Pattillo et al., 1999; Bischofberger et al., 2002; Yang and Wang, 2006). This was tested in a study of somatic calcium currents, in which it was shown that AP broadening at any time during the repolarization phase resulted in an increased amount of calcium influx (Pattillo et al., 1999). Interestingly, a $5 \mathrm{mV}$ hyperpolarization was also tested and found to produce little to no change in the voltage-gated calcium current, which the investigators attributed to a possible balance between the changes of calcium influx and calcium channel gating. This suggests that afterdepolarizations (ADPs) could affect the presynaptic calcium entry and that AHPs may have a small effect or possibly no effect on calcium entry. Given the demonstrated effects on calcium currents, presynaptic afterpotentials could affect neurotransmitter release.

To determine how afterpotentials affect the presynaptic calcium channel currents, we used the calyx of Held nerve terminal, which is located in the auditory brainstem (BarnesDavies and Forsythe, 1995; Borst et al., 1995). The size of this nerve terminal allows presynaptic recordings that can be paired with simultaneous postsynaptic recordings to study the presynaptic and postsynaptic components of synaptic transmission (von Gersdorff and Borst, 2002; Schneggenburger and Forsythe, 2006) Surprisingly, we found that a large range of afterpotentials did not affect the calcium current or neurotransmitter release in this nerve terminal. Our results and simulations of the calcium channel response are consistent with the idea that afterpotentials affect the electrical driving force for calcium entry, but that this is balanced dynamically by the afterpotential effects on calcium channel gating (Pattillo et al., 1999). In addition, this property of voltage-gated calcium channels also acts to prevent or greatly reduce changes in the calcium current that should occur in response to changes in the presynaptic membrane potential. If, for example, the presynaptic membrane potential changes from -80 $\mathrm{mV}$ to $-60 \mathrm{mV}$, the $20 \mathrm{mV}$ difference in the membrane potential at the end of the AP should affect the presynaptic calcium current. However, because the changes in electrical driving force and channel gating cancel each other out, this mechanism greatly reduces the effect that the membrane potential has on the AP-induced calcium current. In summary, this property of calcium channels, in which calcium influx and channel gating can offset each other, serves to greatly reduce or prevent afterpotentials or changes in the presynaptic membrane potential from affecting the release of neurotransmitter and therefore acts to stabilize synaptic transmission.

\section{Materials and Methods}

Brain slice preparation. Brain slices from postnatal day 8 (P8) to P12 CD1 albino mice (Charles River Laboratories) of either sex were used in every experiment described. We also used P8-P12 C57BL/6 mice (Charles River Laboratories) of either sex in some of the dual presynaptic and postsynaptic recording experiments. There were no differences in the experimental results for the two strains of mice. The animals were housed in a facility approved by the Association for Assessment and Accreditation of Laboratory Animal Care International and protocols used for handling and care were reviewed by the Rutgers University Animal Care and Facilities Committee. Slice thickness varied from 150 to $170 \mu \mathrm{M}$. Throughout the process of dissection and slicing, the brain was maintained in a low-calcium artificial CSF (aCSF) solution at $1-2^{\circ} \mathrm{C}$ consisting of the following (in mM): $125 \mathrm{NaCl}, 25 \mathrm{NaHCO}_{3}, 25$ glucose, 3 myoinositol, $2.5 \mathrm{KCl}, 2 \mathrm{Na}$-pyruvate, $1.25 \mathrm{NaH}_{2} \mathrm{PO}_{4}, 0.4$ ascorbic acid, 3 $\mathrm{MgCl}_{2}$, and $0.1 \mathrm{CaCl}_{2}, \mathrm{pH} 7.3$, when oxygenated with carbogen gas $(95 \%$ oxygen, $5 \%$ carbon dioxide). Once produced, slices were transferred to a chamber maintained at $37^{\circ} \mathrm{C}$ for $30-45 \mathrm{~min}$ in normal calcium aCSF solution with the same composition listed above except for $1 \mathrm{~mm} \mathrm{MgCl}$ and $2 \mathrm{mM} \mathrm{CaCl}_{2}$. This same solution was used as our recording solution (see below). Experiments were performed at room temperature for 4-6 $\mathrm{h}$ after the incubation period.

Electrophysiology. To optimize the quality of voltage-clamp and current-clamp recordings, presynaptic terminals were patched at, or in close proximity to, the axonal heminode. Patch-clamp recordings were conducted using an EPC10 USB double patch-clamp amplifier using PatchMaster software (HEKA; Harvard Bioscience). A parasagittal slice orientation was used in all presynaptic voltage-clamp experiments to limit axonal length to minimize space-clamp errors in our recordings. This ensured a more uniform control over the terminal with minimal delay. To help determine the characteristics of the patched cell after recordings, such as axon length and patch location relative to the axon heminode, fluorescent dye $(0.10 \mathrm{mg} / \mathrm{ml}$ Lucifer yellow $)$ was typically added to the presynaptic pipette solutions. For the dual recordings that used current-clamp configuration in the presynaptic terminal, coronal slice orientation was used. Patch pipettes were produced from thickwalled borosilicate glass, $2.0 \mathrm{~mm}$ outer diameter, $1.16 \mathrm{~mm}$ inner diameter (Sutter Instruments). Pipettes with a resistance of 2.5-5 M $\Omega$ were used for presynaptic terminal recordings and pipettes for postsynaptic recordings from principal cells were $2-3.5 \mathrm{M} \Omega$. The series resistance $\left(R_{\mathrm{S}}\right)$ for voltage-clamp recordings was within the range of 5-12 $\mathrm{M} \Omega$ for the presynaptic terminal and $4-10 \mathrm{M} \Omega$ for the postsynaptic cell. An $R_{\mathrm{S}}$ compensation of $60-70 \%$ was applied for all recordings such that the adjusted $R_{\mathrm{S}}$ was in the range of $2-5 \mathrm{M} \Omega$. Bridge compensation of $100 \%$ was used to adjust current injections in current-clamp recordings. Cells that did not meet these criteria were excluded from this study. Sampling frequencies were $200 \mathrm{kHz}$ during presynaptic recordings and $100 \mathrm{kHz}$ for dual recordings. Currents were filtered by a 4 -pole Bessel filter at $3 \mathrm{kHz}$ to remove residual high-frequency noise present in the recordings. Holding potentials were $-80 \mathrm{mV}$ for presynaptic terminals and $-65 \mathrm{mV}$ for postsynaptic cells. A leak subtraction protocol of $\mathrm{P} /-4$ was used to subtract passive effects such as capacitive and leak currents. For presynaptic voltage-clamp recordings of voltage-gated calcium channel activity, the aCSF recording solution was supplemented with tetraethylammonium (TEA; $20 \mathrm{~mm}$ ), tetrodotoxin (TTX; $1 \mu \mathrm{M}$; Tocris Bioscience), and 3,4diaminopyradine (1 mM) to block voltage-gated sodium and potassium channels. The intracellular pipette solution used for measuring presynaptic calcium currents included the following (in $\mathrm{mm}$ ): 90 Csmethansulfonate, $20 \mathrm{CsCl}, 10 \mathrm{TEA}, 40 \mathrm{HEPES}, 1 \mathrm{MgCl}_{2}, 0.5 \mathrm{EGTA}, 5$ phosphocreatine (Alpha Aesar), 2 ATP, and 0.2 GTP, and was buffered to $\mathrm{pH} 7.3$ using $\mathrm{CsOH}$. Intracellular pipette solution for postsynaptic cells during dual recordings consisted of the following (in $\mathrm{mM}$ ): 135 potassium gluconate, $20 \mathrm{KCl}, 10 \mathrm{HEPES}, 5 \mathrm{EGTA}, 5$ phosphocreatine (Alpha Aesar), 2 ATP, and 0.2 GTP and was buffered to $\mathrm{pH} 7.3$ using $\mathrm{KOH}$. Junction potentials, calculated to be $-11 \mathrm{mV}$, were not corrected. For dual recordings in which the presynaptic terminal was in current-clamp configuration, aCSF recording solution without sodium and potassium channel blockers was used and supplemented with bicuculline $(10 \mu \mathrm{M})$ 
and strychnine $(1 \mu \mathrm{M})$ to block inhibitory inputs in the postsynaptic neuron and EGTA was replaced with BAPTA $(0.025 \mathrm{~mm})$ in the presynaptic intracellular solution. Reagents used during all experiments were obtained from Sigma-Aldrich, with the exceptions of TTX, which was obtained from Tocris Bioscience, and phosphocreatine, which was obtained from Alpha Aesar.

To generate fiber-stimulated presynaptic APs, a bipolar platinum stimulating electrode connected to an isolated pulse stimulator (Model 2100; A-M Systems) was placed in contact with the fiber tract at the midline, which contains the axons that form the calyx of Held (Borst et al., 1995). The stimulus consisted of a single $100 \mu$ s stimulation $(\sim 1$ to $20 \mathrm{~V}$ ) to produce each AP. Current-injected APs were generated by a $2 \mathrm{~ms}$ current injection of no more than $500 \mathrm{pA}$ (Kim et al., 2010). To adjust the level of the afterpotential, a hyperpolarizing or depolarizing current injection followed the initial current injection that generated the presynaptic AP. The timing and amplitude of the afterpotential current injection was adjusted as necessary to prevent any changes to the repolarization phase of the AP for the two different afterpotentials. An example of a current injection stimulus is shown in the inset of Figure $4 D$.

Stimulus protocols. Using an AP-like waveform allowed us to more easily test different afterpotential levels. A ramped waveform consisting of a $300 \mu \mathrm{s}$ depolarization followed by a $700 \mu$ s repolarization to the holding voltage was chosen for the standard response. This waveform closely approximated the AP waveforms that were observed in P8-P12 mice and produced calcium current responses that were similar in kinetics and amplitude to those produced by AP stimulation. Afterpotentials were added along the repolarization phase of the AP-like stimuli to study any effect that ADPs and AHPs were having on presynaptic calcium currents. This was performed by ending the repolarization at various potentials in $5 \mathrm{mV}$ increments and maintaining these voltages for a brief period before returning to the holding potential. To preserve the repolarization kinetics of the AP-like stimuli, the duration of the repolarization was adjusted such that afterpotentials were present in a manner that maintained the original slope of the ramp. Experiments were also conducted using real APs stimulated via presynaptic current injection paired with postsynaptic recordings. Hyperpolarizing and depolarizing currents were injected after these APs to produce hyperpolarized potentials of $\sim-100 \mathrm{mV}$ and depolarizations of $-60 \mathrm{mV}$, respectively, to assess the effects that afterpotentials have on the corresponding postsynaptic current.

Data analysis and simulation. Data were analyzed offline using Igor Pro (Wavemetrics) and Excel 2010 (Microsoft). Integrals, peak currents, and durations of presynaptic calcium current responses and excitatory postsynaptic currents (EPSCs) were measured using Igor Pro with the unipolar peak/area detection procedure and a trapezoidal approximation method. Peak currents were measured relative to the baseline of the data traces. Our measurements required stable recordings to accurately determine small differences in the presynaptic calcium currents. Therefore, if the response to the same stimulation was fluctuating $>5 \%$, we continued to stimulate for several times until the response stabilized. If this did not occur, then the data traces from that cell were not included in the analyzed dataset. To align the traces for accurate measurement, a constant was subtracted from individual traces to align the baseline to zero by visual inspection. Presynaptic calcium integrals were measured from the onset of the inward calcium current to the point at which the current returned to the baseline of the data trace. EPSC integrals were measured from the onset of the response to the point at which the current returned to baseline levels or measured in $1 \mathrm{~ms}$ increments for a total of $10 \mathrm{~ms}$ to assess the currents during both peak activity and during the return to baseline. This approach was used to determine whether there was any change early on in the EPSC response and to measure any differences in early or later portions of the response. The data were collected for comparative and statistical analysis using Microsoft Excel. Data values are expressed as the mean \pm the SEM of the experimental sets being analyzed unless otherwise stated. Statistical significance was used to compare the responses to an ADP with responses to an AHP and was assessed using Student's $t$ test: two-tailed, two samples with equal variance $\left({ }^{*} p<0.05\right.$; $\left.{ }^{\star *} p<0.01 ;{ }^{* *} p<0.005\right)$. Simulations of the macroscopic voltage-gated calcium current were calculated numerically by the Euler integration method using intervals of $100 \mathrm{nsec}$. Rate constants were adjusted manually to optimize the fit of the simulated response to an AP-like stimula- tion with recorded presynaptic calcium channel responses to an AP-like stimulation ending in an AHP or ADP. Voltage-dependent rate constants in the forward direction are in the format of $\alpha_{i}(V)$ and backward rates are $\beta_{\mathrm{i}}(V)$. The microscopic rate constants were exponentially dependent on voltage as described by the following equations: $\alpha_{\mathrm{i}}(V)=\alpha_{\mathrm{io}} \exp \left(V / V_{\mathrm{i}}\right)$ and $\beta_{\mathrm{i}}(V)=\beta_{\mathrm{io}} \exp \left(-V / V_{\mathrm{i}}\right)$.

The gating scheme and the rate constants used for the simulations are shown in Figure 6E.

\section{Results}

\section{Afterpotentials after instant voltage jumps affect neurotransmission}

Afterpotentials, which occur near the end of the AP repolarization phase, should affect neurotransmitter release by affecting the electrical driving force for calcium entry and altering the kinetics of the voltage-gated calcium channel response. To determine whether afterpotentials affect synaptic transmission, we used the calyx of Held nerve terminal, which allows simultaneous presynaptic and postsynaptic patch-clamp recordings and has been used previously to determine the effects of AP kinetics on calcium channel response and neurotransmitter release (Borst and Sakmann, 1999; Wu et al., 2004; Yang et al., 2014). After blocking voltage-gated sodium and potassium channels (see Materials and Methods), the response to the presynaptic voltage-gated calcium channels can be measured.

Previous experiments using instant voltage jumps to activate presynaptic voltage-gated calcium channels have shown that afterpotentials affect both the amplitude and the deactivation kinetics of the calcium channel tail current response (Borst and Sakmann, 1999; Bischofberger et al., 2002). To determine how these changes in the presynaptic calcium channel response affect neurotransmitter release, we performed simultaneous presynaptic and postsynaptic patch-clamp recordings (Fig. $1 A, B$ ). In agreement with the results mentioned above, we found that the amplitude of the presynaptic calcium channel response increases due to the increased electrical driving force for calcium entry as the afterpotential becomes more negative (Fig. $1 A$, inset). In addition, the time course of deactivation becomes faster as the afterpotential becomes more negative due to the voltage-dependent gating of calcium channels (Fig. 1A, inset). Interestingly, in the simultaneous postsynaptic recordings, the presynaptic voltage jump that ended with an ADP produced the largest postsynaptic response (Fig. $1 A-C$ ). Although the ADP stimulus produced a presynaptic calcium channel response with a smaller peak amplitude (Fig. 1A), it also has slower deactivation kinetics, which results in a greater amount of calcium entry and therefore a larger amount of neurotransmitter release. This further demonstrates that the total influx of calcium is more important than the peak amplitude in determining the amount of neurotransmitter release (Yang and Wang, 2006).

To determine whether afterpotentials affect the response to repeated stimulation, we applied a train of four stimuli at $100 \mathrm{~Hz}$ (Fig. $1 B, C$ ). For the four stimuli, the postsynaptic response to a presynaptic ADP was larger than the response to a presynaptic AHP (Fig. $1 C$, responses 1-4: $p<0.001 ; t$ test, $n=5$ simultaneous presynaptic and postsynaptic recordings). We found that the peak amplitude of the calcium current was most notably different for the first stimulus, in which the peak of the current generated by an AHP was greater than that generated by an ADP (Fig. 1D). For the next three stimuli in the train, the peak amplitude of the calcium current in response to an AHP was consistently higher than the peak amplitude generated by ADP, but the differences were not found to be statistically significant. In contrast, the amount of calcium entry measured by the integral of the presynaptic calcium current (Fig. 1E) was consistently larger in response 
A

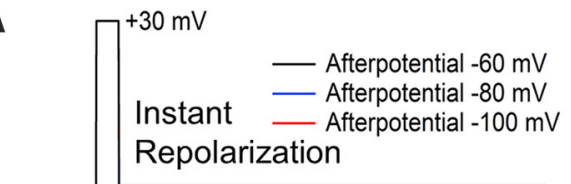

$-80 \mathrm{mV}$
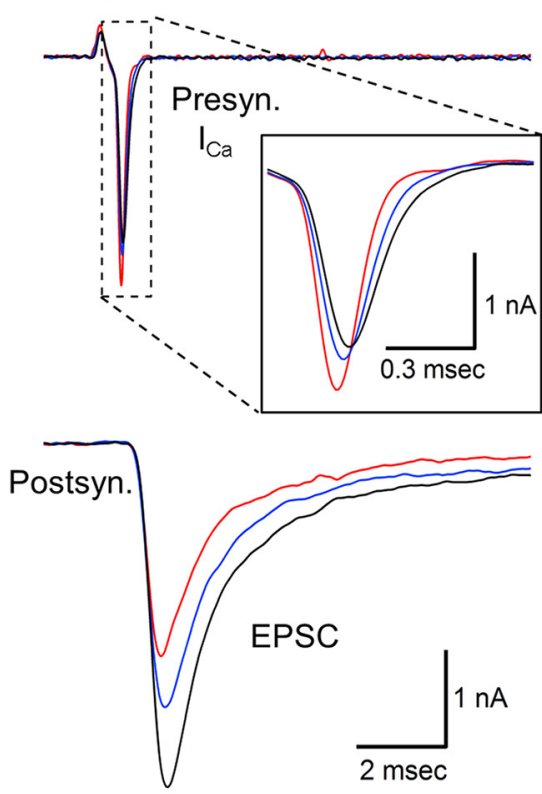

D

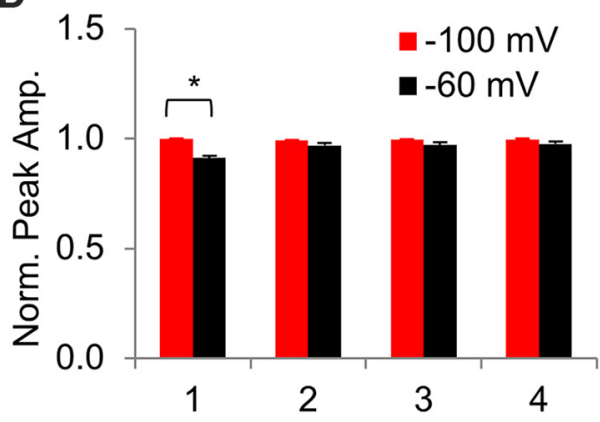

EPSC Stimulation Number
B

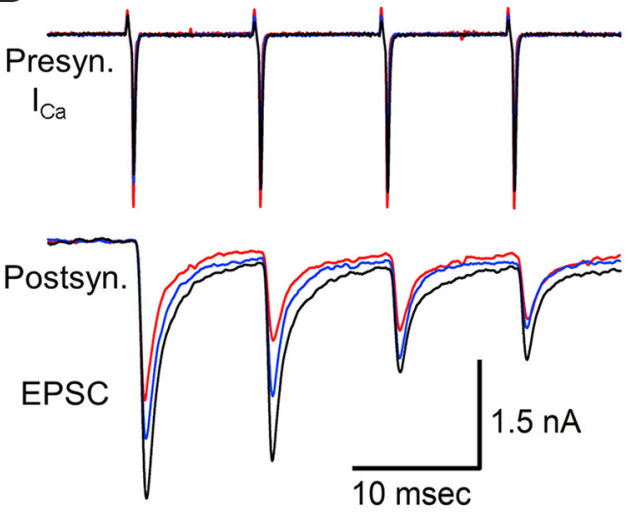

C

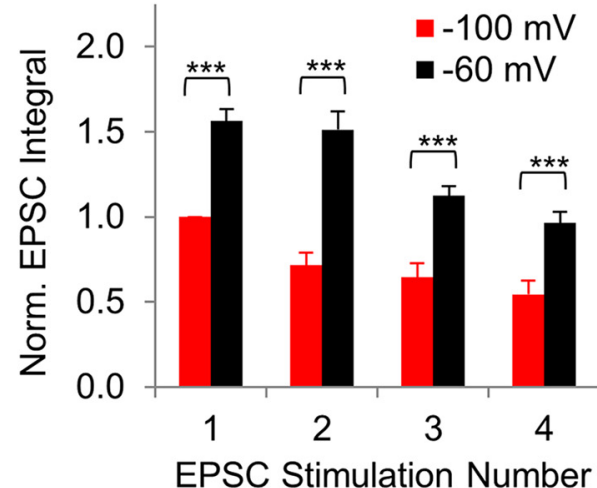

E

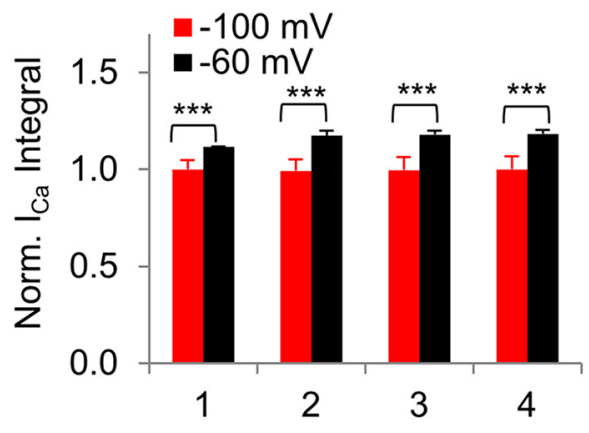

EPSC Stimulation Number

Figure 1. Afterpotentials after an instant voltage jump affect the presynaptic calcium current and the postsynaptic response. $A$, In simultaneous presynaptic and postsynaptic recordings, a 0.5 ms voltage jump was used to activate presynaptic voltage-gated calcium channels. After the voltage jump, the membrane potential was returned to $-60 \mathrm{mV},-80 \mathrm{mV}$, or $-100 \mathrm{mV}$ to determine the response to an ADP (black), the normal response (blue), or an AHP (red), respectively. The center trace shows an overlay of the presynaptic calcium current. The bottom trace shows the simultaneously recorded excitatory postsynaptic current (EPSC). $B$, Presynaptic calcium channel response to a train of $0.5 \mathrm{~ms}$ voltage jumps at $100 \mathrm{~Hz}$ with simultaneously measured postsynaptic responses. C, The area of each postsynaptic response was measured to determine how synaptic transmission is affected by presynaptic afterpotentials that follow voltage jumps. EPSC integral is expressed as mean, with error bars indicating the SEM in response to a $-100 \mathrm{mV}$ afterpotential (red) or a $-60 \mathrm{mV}$ afterpotential (black). The postsynaptic response to presynaptic voltage jump afterpotentials of $-100 \mathrm{mV}$ and $-60 \mathrm{mV}$ showed a highly significant difference at each stimulation ( ${ }^{* * *} p<0.005, t$ test, $n=5$ paired presynaptic and postsynaptic recordings). D, Normalized peak amplitude of the presynaptic calcium current with SEM error bars. Responses are normalized to the amplitude of the first response to a stimulus train with $-100 \mathrm{mV}$ AHPs $\left({ }^{*} p<0.05, t\right.$ test, $n=5$ paired presynaptic and postsynaptic recordings). $\boldsymbol{E}$, Normalized average area of the presynaptic calcium currents with error bars indicating SEM. Responses are normalized to the first response to the voltage jump with a $-100 \mathrm{mV}$ afterpotential. The responses to an afterpotential at $-100 \mathrm{mV}$ versus $-60 \mathrm{mV}$ showed a highly significant difference at each stimulation $\left({ }^{* * *} p<0.005, t\right.$ test, $n=5$ paired presynaptic and postsynaptic recordings).

to a voltage-jump ADP compared with responses generated by an AHP. These findings indicate that the total calcium influx is more important than the peak amplitude of the calcium current in determining the amount of neurotransmitter release.

Our results show a steady decline in the postsynaptic response to a presynaptic AHP (Fig. $1 C$, red). The postsynaptic response to a presynaptic $\mathrm{ADP}$ also shows a steady decline, but it appears to occur after the second stimulus (Fig. $1 C$, black). A likely explanation for this is that there is a slight increase in the size of the calcium current, starting with the second presynaptic ADP stimulus (Fig. 1E). Stimulation at high frequency can result in a calcium-dependent facilitation of the calcium channel response and facilitation is sensitive to slight differences in calcium influx (Lin et al., 2012), which could explain the lack of facilitation in 
the calcium current generated by the AHP stimuli (Fig. 1E, red). Aside from the second postsynaptic response, there is a steady decline in the postsynaptic response generated by a train of presynaptic voltage-jump stimuli that are followed by an ADP.

In summary, these data demonstrate that afterpotentials after a standard instant voltage jump affect both the amplitude and deactivation of the calcium channel response (Fig. $1 A, B$ ), which then affects the release of neurotransmitter and the postsynaptic response (Fig. $1 A-C$ ). For presynaptic voltage-jumps, we find that the effects of afterpotentials on calcium channel gating are more important than the effects on electrical driving force in determining the postsynaptic response.

\section{Afterpotentials after an AP-like stimulus have a minimal effect on the presynaptic calcium current}

To study how afterpotentials affect the calcium channel response to a more physiological stimulus, we used AP-like stimulations consisting of a ramped $0.3 \mathrm{~ms}$ depolarization from $-80 \mathrm{mV}$ to $+60 \mathrm{mV}$ followed by a $0.7 \mathrm{~ms}$ repolarization, which closely matched the kinetics and the duration of APs produced by fiber stimulation and produced a response similar to ones generated by recorded APs. This allowed us to add afterpotentials of various amplitudes and durations. Ramped AP-like stimuli have been used previously to activate presynaptic calcium channels and determine how changes in the AP waveform affect synaptic transmission (Yang and Wang, 2006; Chen et al., 2015; Han et al., 2015).

Using the AP-like stimulus, we first wanted to determine the portion of the voltage-gated calcium channel response that could be affected by afterpotentials. The exact timing of the calcium channel response with respect to the timing of the AP-like stimulus is difficult to determine because calcium channels do not begin to activate until $\sim-40 \mathrm{mV}$ and the initial activity is obscured by a brief upward deflection in the calcium channel response that is thought to be due to a gating current (Stanley and Goping, 1991; Borst and Sakmann, 1998). To better understand the relationship between the APlike stimulus and the calcium channel response and to determine the amount of calcium channel activity that is still present when afterpotentials occur, we used both depolarizing voltage jumps to $+60 \mathrm{mV}$ and hyperpolarizing voltage jumps to $-150 \mathrm{mV}$ that were given when the AP repolarization reached $-60 \mathrm{mV}$. Both depolarizing and hyperpolarizing voltage jumps produced an inflection in the calcium channel response. We used the onset of the inflection as a marker to determine how the timing of the stimulus correlates with the timing of the calcium channel response. This allowed us to determine that a large portion of the calcium channels are still active at the onset of the ADP (Fig. $2 A$ ). To determine the percentage of the calcium current that occurs after the onset of the afterpotential, we measured the area of the calcium current that occurs after the onset of the afterpotential and compared that with the total area of the calcium current. We found that $48.5 \%$ of the calcium current occurs after the onset of the -60 $\mathrm{mV}$ afterpotential ( $\pm 2.5 \% \mathrm{SEM}, n=11$ calyces $)$. Based on the timing of the voltage jumps, we conclude that afterpotentials could affect a large portion of the calcium channel response to an AP-like stimulus.

To study the effects of afterpotentials, we set our ADP level at $-60 \mathrm{mV}$, which matches the peak of a typical ADP in this nerve terminal (Paradiso and $\mathrm{Wu}, 2009$; Kim et al., 2010) and corresponds to a $20 \mathrm{mV}$ depolarization from the holding potential. We then set our AHP at $-100 \mathrm{mV}$, which is equal and opposite in amplitude to the ADP when the holding potential is at $-80 \mathrm{mV}$. APs from several types of neurons display afterpotentials that occur within this range (Bean, 2007).

Surprisingly, the calcium channel response was not noticeably affected by the two different afterpotentials despite a $40 \mathrm{mV}$ difference in the membrane potential at the end of the stimulus (Fig. $2 B$ ). Therefore, in contrast to the results when instant voltage jumps were used to activate calcium channels, the response to a ramped repolarization does not appear to be affected by afterpotentials. To better resolve this, we averaged 10 calcium channel responses produced by a $-60 \mathrm{mV} \mathrm{ADP}$ and subtracted this from the average of 10 calcium channel responses generated by the $-100 \mathrm{mV}$ AHP (Fig. 2B, cyan trace). The subtracted average trace shows that the calcium current produced by an AHP has a maximum amplitude of only $43 \mathrm{pA}$ of additional current compared with the response generated by the ADP in this calyx (Fig. $2 B$, inset). We repeated this in several nerve terminals and then divided the area of the subtracted trace from the area of the trace produced by an AHP and found a brief current that was $<3 \%$ of the total calcium influx $($ mean $=2.4 \%, \mathrm{SEM}=1.2 \%, n=6$ nerve terminals). In contrast to the instant voltage jumps, the calcium channel response to a ramped repolarization is relatively unaffected by afterpotentials.

This result prompted us to determine how large the amplitude of an ADP must be before it produces a visible difference in the presynaptic calcium channel response to an AP-like stimulation. We therefore tested a larger range of ADP amplitudes to values that far exceed the physiological range of the ADP present at the calyx of Held (Fig. 2C). Our results show that afterpotentials below $-40 \mathrm{mV}$ do not appear to alter the calcium channel response. At $-35 \mathrm{mV}$, there is a slight delay in the decay of the calcium current and a prolonged residual calcium current is present at the end of the calcium channel response (cyan trace). As the ADP level increases, the amount of residual activity steadily increases. This eventually produces a prolonged, large amplitude calcium current. However, voltage-gated calcium channels in the presynaptic terminal begin to activate $\sim-40 \mathrm{mV}$, suggesting that the responses we are seeing with ADPs above $-40 \mathrm{mV}$ are simply due to the expected level of calcium channel activation that would normally occur in the absence of a preceding AP-like stimulation. To test this, we terminated the response from the AP-like stimulation by adding a $1 \mathrm{~ms}$ interval before the onset of the afterpotential (Fig. 2D) to separate the AP-like response from the afterpotential responses. Comparison of the traces shows a similar amplitude and time course in the presence or absence of the 1 ms separation period. This indicates that the prolonged activity that is apparent with ADPs above $-40 \mathrm{mV}$ appears to be largely independent of the calcium channel response to the AP-like stimulation. Therefore, for ADPs, the calcium channel response to an AP-like stimulation only changes when the ADP reaches a membrane potential at which calcium channel activation begins. These results further demonstrate that physiological amplitudes of afterpotentials have a minimal effect on the presynaptic calcium channel current.

\section{Changes in the presynaptic membrane potential from -60 to $-100 \mathrm{mV}$ have a minimal effect on the presynaptic calcium current}

The resting potential of presynaptic terminals can change briefly due to the activity of electrogenic pumps and transporter currents (Meir et al., 1999; Kim et al., 2007; Paradiso and Wu, 2009), presynaptic ionotropic receptors (MacDermott et al., 1999; Turecek and Trussell, 2001), and analog signals arriving from the 

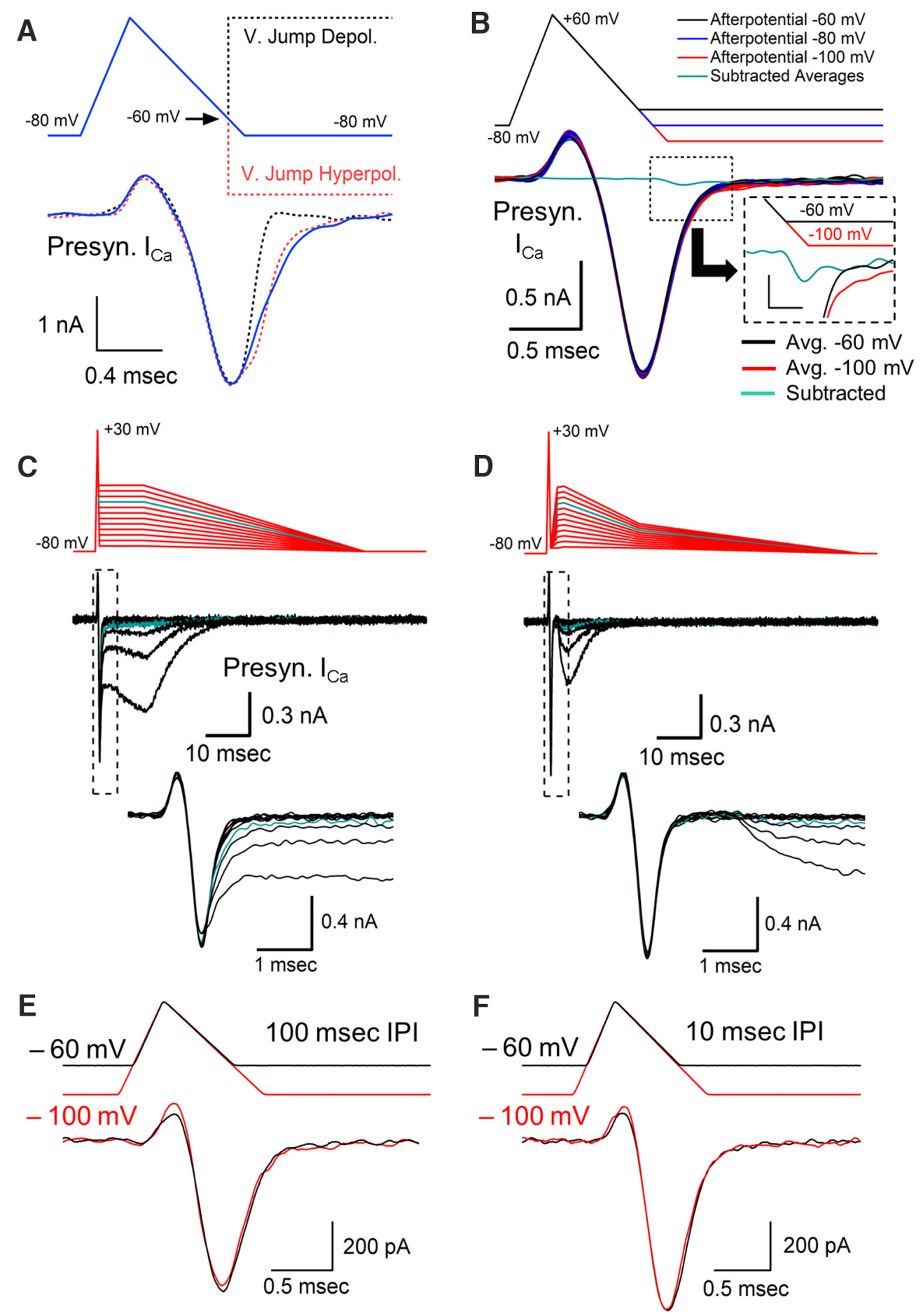

Figure 2. Effects of afterpotentials after an AP-like stimulus. A, Depolarizing voltage jumps to $+60 \mathrm{mV}$ and hyperpolarizing voltage jumps to $-150 \mathrm{mV}$ were used during an AP-like stimulation to determine the amount of calcium channel activity that is present at the time that our standard afterdepolarization occurs. B, Responses to AP-like stimulation with afterpotentials at $-60,-80$, and $-100 \mathrm{mV}$. Responses are from a representative cell, with each value tested 10 times. Cyan trace shows the average of 10 calcium channel responses to a $-60 \mathrm{mV}$ afterpotential subtracted from the average of 10 responses to a $-100 \mathrm{mV}$ afterpotential. Inset shows subtracted average trace at higher resolution with the stimulus bar shifted $0.15 \mathrm{~ms}$ to adjust for the delay between the stimulus and the response. Inset scale bars, $50 \mathrm{pA}, 0.3 \mathrm{~ms}$. C, Effects of ADPs on presynaptic voltage-gated calcium current over a range of -75 to $-20 \mathrm{mV}$ tested in $5 \mathrm{mV}$ increments. Cyan line $(-35 \mathrm{mV})$ indicates afterpotential amplitude where calcium current is first affected. Bottom trace shows calcium current at higher temporal resolution. $\boldsymbol{D}$, Effects of separating the afterpotential from the AP waveform by returning the membrane potential to $-75 \mathrm{mV}$ for $1 \mathrm{~ms}$ followed by afterpotentials over a range of -75 to $-20 \mathrm{mV}$ tested in $5 \mathrm{mV}$ increments. Cyan line $(-35 \mathrm{mV})$ indicates potential amplitude where calcium channels begin to respond. $\boldsymbol{E}$, Changing the membrane potential has only a small effect on the presynaptic calcium current generated by an AP-like stimulation. Overlay of two responses from a $10 \mathrm{~Hz}$ stimulation train. The membrane potential was held at $-60 \mathrm{mV}$ (black trace) or $-100 \mathrm{mV}$ (red trace) for $100 \mathrm{~ms}$ before the AP-like stimulus. A small difference in the transient upward current is apparent between the responses at -60 $\mathrm{mV}$ compared with $-100 \mathrm{mV}$, consistent with differences that would be produced by a gating charge. Trains were $<0.5 \mathrm{~s}$ in duration. $\boldsymbol{F}, 0$ verlay of two responses occurring during a 100 $\mathrm{Hz}$ stimulus train. The membrane potential was at $-60 \mathrm{mV}$ (black trace) or $-100 \mathrm{mV}$ (red trace) for $10 \mathrm{~ms}$ before the AP-like stimulus. 
axon (Alle and Geiger, 2006; Shu et al., 2006). We hypothesized that the same mechanism that stabilizes synaptic transmission in response to afterpotentials should also stabilize calcium channel responses if the membrane potential changes. Accordingly, we measured the effects of delivering an AP-like stimulation at different resting potentials. For these experiments, we used brief trains of AP-like stimuli, $0.5 \mathrm{~s}$, applied at $10 \mathrm{~Hz}$. The membrane potential was held at either $-100 \mathrm{mV}$ (Fig. $2 E$, red traces) or at $-60 \mathrm{mV}$ (Fig. 2E, black traces) between stimuli and the resulting calcium currents show little or no difference in their response. Due to the change in membrane potential, one concern was that steady-state inactivation could be at least partially responsible for this effect. However, a large contribution is unlikely because voltage-gated calcium channels in the calyx of Held show very little steady-state inactivation after a $500 \mathrm{~ms}$ voltage step (Forsythe et al., 1998; Lin et al., 2011), particularly over the range of $-100 \mathrm{mV}$ to $-60 \mathrm{mV}$, where steady-state inactivation is $<10 \%$ (Forsythe et al., 1998). To further verify this, we repeated our experiment with stimulus trains at $100 \mathrm{~Hz}$. If steady-state inactivation were affecting our results, then we would expect that the 10 -fold increase in the duration of the membrane potential change should have a noticeable effect on the response, but we did not observe an appreciable difference (Fig. $2 F$ ). The ratio of the calcium currents generated at a depolarized membrane potential to the response generated at a hyperpolarized membrane potential for the $100 \mathrm{~Hz}$ trains is $1.01 \pm 0.03 \mathrm{SEM}(n=10)$ compared with $1.04 \pm 0.03 \mathrm{SEM}(n=4)$ for the $10 \mathrm{~Hz}$ trains. This indicates that steady-state inactivation is not playing an appreciable role. Because the majority of calcium entry occurs during the repolarization phase of the AP-like stimulus, it is likely that the mechanism that minimizes the effects of afterpotentials also limits any difference in the calcium current from the stimuli delivered at the two different membrane potentials.

\section{Afterpotentials after AP-like stimuli do not affect neurotransmission}

It is possible that small differences in the presynaptic calcium channel response could still affect synaptic transmission. For example, changes in calcium influx that are not easily detectable by direct comparison of the traces could still affect synaptic transmission (Awatramani et al., 2005). Small differences in calcium entry can be detected by changes in the postsynaptic response because neurotransmitter release is highly sensitive to small fluctuations in the local internal calcium concentration due to nonlinear calcium sensitivity (Bollmann et al., 2000; Schneggenburger and Neher, 2000; Fernández-Chacón R et al., 2001; Eggermann et al., 2012). Postsynaptic recordings can also be used to directly test whether afterpotentials affect synaptic transmission. Therefore, we used simultaneous presynaptic and postsynaptic recordings to detect any changes in neurotransmitter release in response to presynaptic afterpotentials. In agreement with our earlier presynaptic recordings, the presence of an AHP or ADP did not appear to affect the presynaptic calcium channel response in the paired recordings (Fig. 3A). In addition, the release of neurotransmitter measured by the simultaneous postsynaptic recordings also did not show a statistically significant difference when comparing the postsynaptic current in response to a presynaptic AHP or ADP (Fig. 3A). The postsynaptic response to a presynaptic ADP was very similar to the normalized response to an AHP $(0.99 \pm 0.9 \mathrm{SE}, p=0.9, n=6$ simultaneous presynaptic and postsynaptic recordings). To determine whether an afterpotential after an AP-like stimulus could affect the responses to repeated stimulation, as they do for the instant voltage jumps
(Fig. 1C), we applied a train of four stimuli at $100 \mathrm{~Hz}$ (Fig. 3B-D). Even with repeated stimuli, we do not find a difference in the presynaptic calcium current in response to an afterdepolarization or an afterhyperpolarization (Fig. 3B, C). For the four stimuli, although the postsynaptic response to a presynaptic ADP was slightly larger than the response to an AHP, the differences were not statistically significant (Fig. $3 D, p=0.84,0.54,0.29$ and 0.24 , in order for the first to fourth stimulus, $t$ test, $n=5$ simultaneous presynaptic and postsynaptic recordings).

We also note that, at high frequencies, an afterpotential can be present for the entire duration during the interstimulus interval (Paradiso and Wu, 2009; Kim et al., 2010). To replicate what happens physiologically, the afterpotentials in our repeated stimulation experiment were present for the entire duration between stimuli (Fig. 3B). We note that this is effectively a change in the membrane potential to $-60 \mathrm{mV}$ (Fig. $3 B$, black) or $-100 \mathrm{mV}$ (Fig. $3 B$, red) for the second, third, and fourth stimuli. This demonstrates that a change in the membrane potential shortly before an AP-like stimulation does not affect the presynaptic calcium current or the postsynaptic response.

\section{Afterpotentials after real presynaptic APs do not affect the postsynaptic response}

The AP-like stimuli used to study afterpotentials have kinetics that are a reasonable approximation of an AP stimulus, but slight differences in the kinetics of a real AP versus an AP-like stimulus could affect the calcium channel response. Therefore, as a final method for testing whether afterpotentials can affect synaptic transmission, in a separate set of experiments in the absence of blockers, we used real APs stimulated presynaptically in currentclamp mode while simultaneously recording the postsynaptic response in voltage-clamp mode. APs generated in the calyx of Held nerve terminal exhibited a fast AHP (Kim et al., 2010) that reaches $\sim-65$ to $-75 \mathrm{mV}$, followed by an ADP that peaks at $\sim-60 \mathrm{mV}$ (Fig. $4 A, C$, top traces, black). In addition, during repeated stimulation, the fast AHP level can change. For example, at $100 \mathrm{~Hz}$, the fast AHP changes $\sim 5 \mathrm{mV}$ over a $1 \mathrm{~s}$ stimulation period (Fig. 4A). To study how different afterpotentials would affect neurotransmitter release, we used current injections to alter the existing afterpotentials. Presynaptic APs were generated by a brief, $2 \mathrm{~ms}$, depolarizing current injection in current-clamp mode. APs generated by current injection were very similar to those generated by fiber stimulation, particularly in the repolarization phase (Fig. 4B). After the initial depolarizing current injection, a small depolarizing current injection was applied to ensure that the fast AHP amplitude reached $-60 \mathrm{mV}$ or a hyperpolarizing current injection was used to create a slow AHP similar to ones that occur physiologically in other types of neurons (Fig. $4 D$, inset). Although this experiment does not allow the measurement of the presynaptic calcium current, the postsynaptic responses (Fig. $4 C, D$, bottom traces) provide a sensitive measurement of any changes in presynaptic calcium influx and addresses directly whether presynaptic APs are capable of affecting synaptic transmission.

In agreement with the results using the AP-like stimulation, the experiments using real presynaptic APs with modified afterpotentials did not demonstrate a difference in the postsynaptic response when the APs ended in either an ADP or an AHP (Fig. $4 C-E)$. This was quantified by measuring the integral of each postsynaptic response generated by a $100 \mathrm{~Hz}$ train of 10 presynaptic APs (Fig. 4C,D). Our voltage-jump experiments (Fig. 2A) show that approximately half of the calcium entry occurs before the AP reaches $-60 \mathrm{mV}$. Therefore, this would likely affect the 

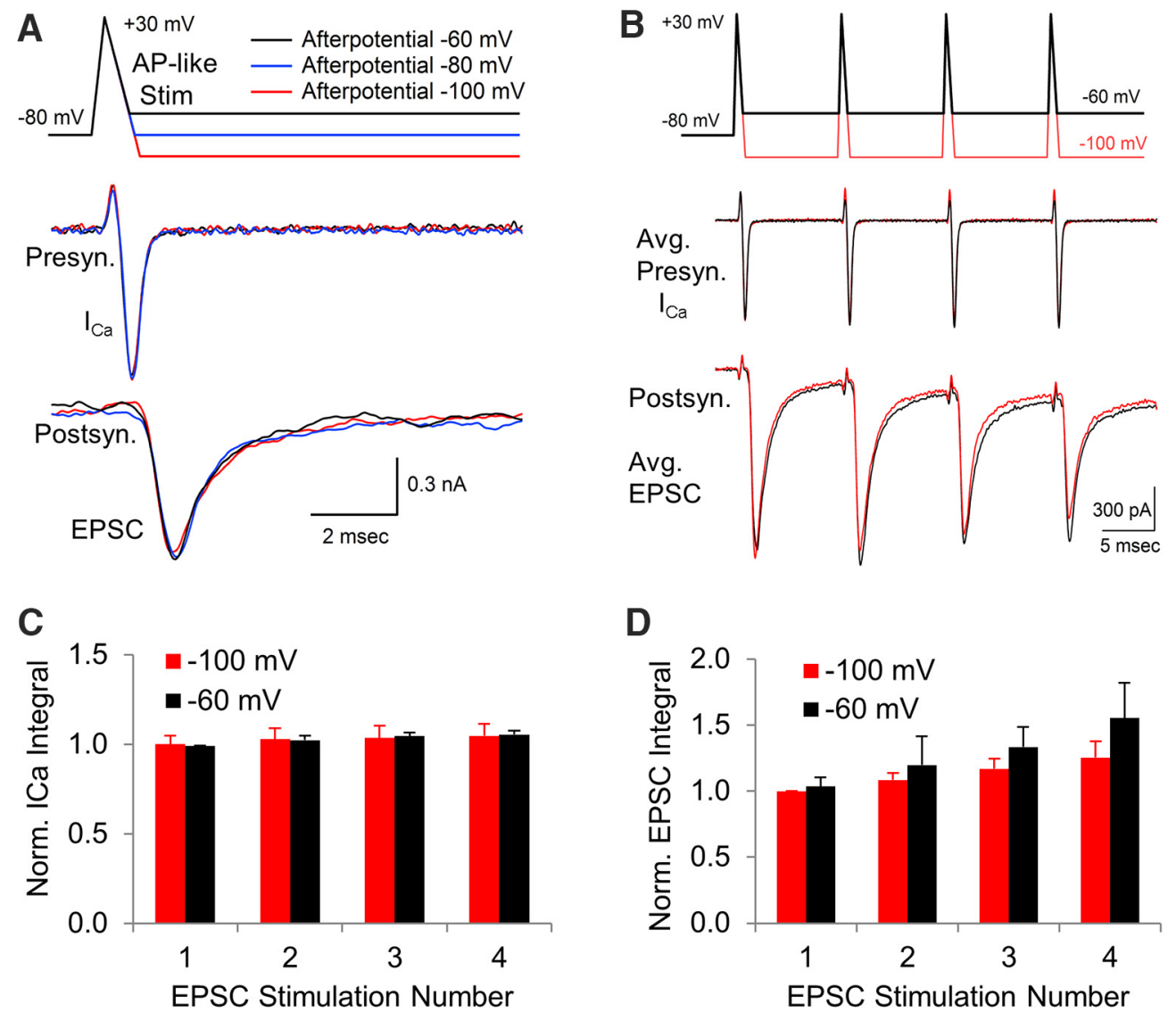

Figure 3. Simultaneous presynaptic and postsynaptic recordings to determine whether afterpotentials after AP-like stimuli affect synaptic transmission. $\boldsymbol{A}$, Simultaneous presynaptic and postsynaptic recordings in response to a presynaptic AP-like stimulus used to activate voltage-gated calcium channels. During the repolarization phase, the membrane potential change ended at $-60 \mathrm{mV},-80 \mathrm{mV}$, or $-100 \mathrm{mV}$ to determine the response to an ADP (black), a normal response (blue) or an AHP (red), respectively. Center trace shows an overlay of the presynaptic calcium current. Bottom trace shows the EPSC in response to presynaptic afterpotentials. $\boldsymbol{B}$, To determine whether afterpotentials affect calcium buildup between APs, we tested the presynaptic calcium channel response to a train of AP-like stimuli with an ADP ( $-60 \mathrm{mV}$, black) or an AHP ( $-100 \mathrm{mV}$, red) tested at $100 \mathrm{~Hz}$ while simultaneously measuring the postsynaptic responses. The postsynaptic responses to a presynaptic ADP versus an AHP showed no significant difference at each stimulation. C, Average area of the presynaptic calcium currents. Error bars indicate SEM. Responses are normalized to the first response to an AP-like stimulation with a $-100 \mathrm{mV}$ afterpotential. The small differences in the responses to an afterpotential at $-100 \mathrm{mV}$ versus $-60 \mathrm{mV}$ are not statistically significant ( $p>0.5, t$ test, $n=5$ paired presynaptic and postsynaptic recordings). $D$, Normalized area (mean, with SEM error bars) of each postsynaptic response generated by four presynaptic AP-like stimuli followed by an ADP ( $-60 \mathrm{mV}$, black) or an AHP $(-100 \mathrm{mV}$, red) showed no significant difference at each stimulation ( $p>0.2, t$ test, $n=5$ paired presynaptic and postsynaptic recordings). EPSC integral is expressed as mean, with error bars indicating SEM.

timing of any effect of afterpotentials on the postsynaptic response. Small differences occurring in only a portion of the postsynaptic response could be difficult to detect by measuring the integral of the entire response. Accordingly, we separately measured the integral for each millisecond of the postsynaptic response (Fig. 4D,E), giving a total of 10 measurements for each postsynaptic response and 100 measurements for each train of 10 responses. The similarity between each set of measurements provides further proof that the presence of an ADP or AHP did not affect the postsynaptic response ( $p>0.1, t$ test, $n=7$ simultaneous presynaptic and postsynaptic recordings; Fig. $4 C-E)$. This also further supports the finding that a change in the resting potential over a range that afterpotentials can produce does not affect neurotransmitter release because, after the first AP in the train, the subsequent APs are generated from a hyperpolarized or depolarized value compared with the initial membrane potential (Fig. 4C).

\section{Physiological repolarization eliminates the effects of} afterpotentials on calcium channel currents

The results described above demonstrate a discrepancy between the responses from presynaptic calcium channels activated by instant voltage jumps versus APs. We pursued this further by testing an instant repolarization (Fig. $5 A$, left trace) and then compared this response with responses generated by a stimulus with a 0.5 or $1 \mathrm{~ms}$ ramped repolarization (Fig. $5 A$, center and right traces). To maximize the calcium channel response, a $10 \mathrm{~ms}$ depolarization was used to activate the calcium channels present in the nerve terminal fully. We found that the changes in peak amplitude and deactivation rate that occur when an instant repolarization ends in an afterpotential were largely reduced when the repolarization phase was increased to $0.5 \mathrm{~ms}$ and were nearly absent with a $1 \mathrm{~ms}$ repolarization (Fig. $5 A$ ). Because the depolarization portion was the same for all three stimuli, the effects on peak amplitude and timing are due to the differences in the repolarization rates. Next, we tested a shorter voltage jump and compared this response with responses generated by AP-like stimulations with a $0.5 \mathrm{~ms}$ repolarization or a $0.7 \mathrm{~ms}$ repolarization (Fig. $5 B$ ). For the instant repolarization, the response to an afterpotential of $-100 \mathrm{mV}$ was compared with the response from an afterpotential at $-50 \mathrm{mV}$, which shows an $\sim 90 \mu$ s increase in the timing of the peak amplitude of the calcium channel response (Fig. 5C). The instant repolarization also displays a steady decrease in peak amplitude that occurs with afterpotentials from -100 to 
A

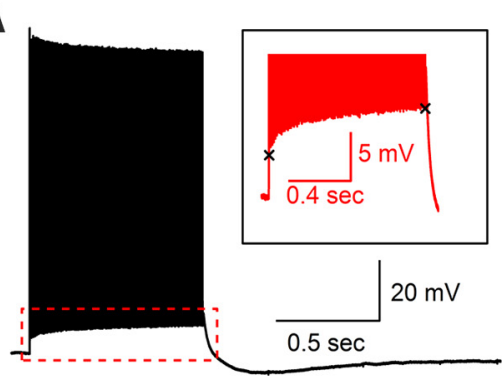

B

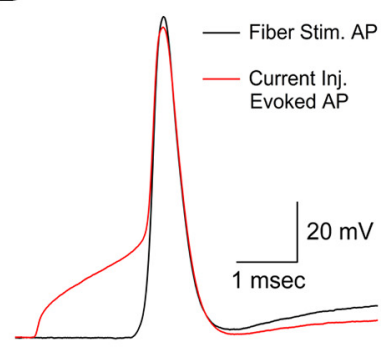

C

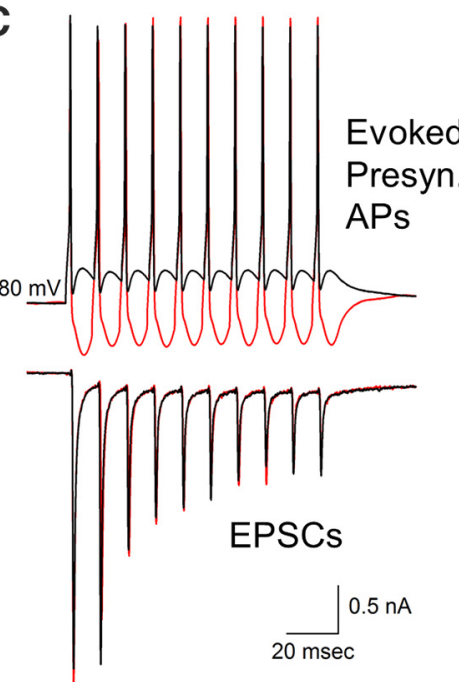

D

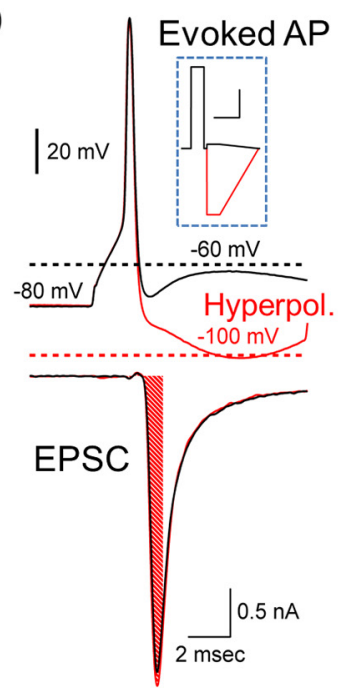

$E$ Postsynaptic Responses at $1 \mathrm{msec}$ Intervals

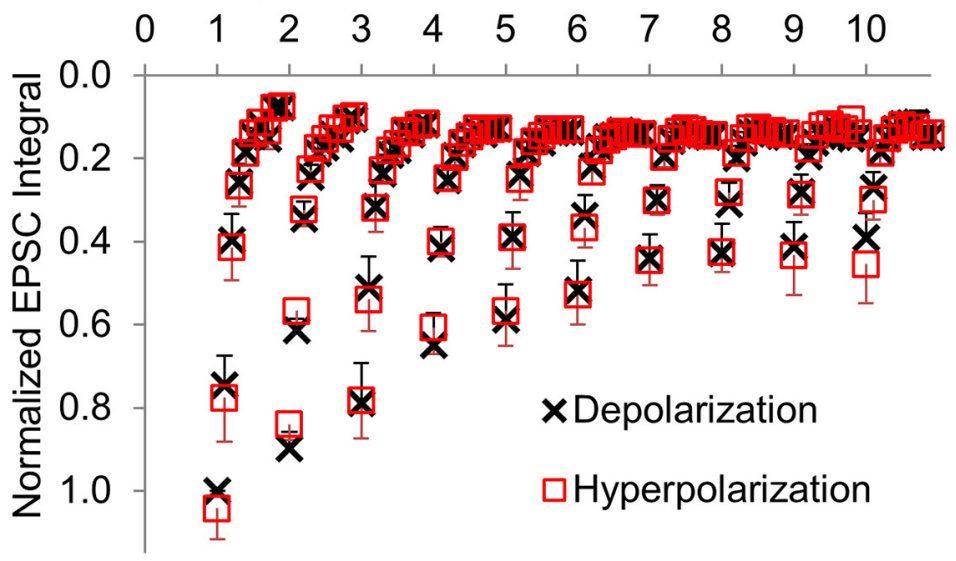

Figure 4. Postsynaptic effects of real presynaptic APs with altered afterpotentials. A, Train of APs generated by stimulating the fiber tract at $100 \mathrm{~Hz}$ for $1 \mathrm{~s}$ showing that the response is elevated by the ADP. Inset shows an $\sim 5 \mathrm{mV}$ change in the ADP during the $1 \mathrm{~s} \mathrm{stimulation} \mathrm{period.} \boldsymbol{B}$, Presynaptic APs generated by a brief current injection (red trace) are very similar to the APs generated by fiber stimulation (black trace). C, Presynaptic APs were generated by a brief depolarizing current injection to reach threshold, followed by a current injection to create an AHP or alter the amplitude of the ADP. $\boldsymbol{D}$, Higher resolution of first response shown in A. The bottom trace shows an example of an area measurement over a $1 \mathrm{~ms}$ interval in the EPSC response. Inset shows a typical stimulus used to generate the presynaptic AP and change afterpotential levels. Inset scale bars, $4 \mathrm{~ms}, 200 \mathrm{pA}$. $\boldsymbol{E}$, To resolve small differences throughout the response, the area of each postsynaptic response was measured in $1 \mathrm{~ms}$ increments for a total of 10 measurements for each postsynaptic response. The 1 ms postsynaptic area measurements (mean, with SEM error bars, $n=7$ ) in response to presynaptic APs with an AHP versus an ADP are overlaid for comparison.

$-50 \mathrm{mV}$ (Fig. $5 D$ ). In contrast, for the 0.5 and $0.7 \mathrm{~ms}$ repolarization, the timing $(p<0.001, t$ test compared to instant repolarization, $n=$ 9; Fig. $5 C$ ) and amplitude of the peak calcium channel response $(p<$ $0.05, t$ test compared to instant repolarization, $n=9$; Fig. $5 D)$ are

minimally affected by afterpotentials over the range of -100 to $-50 \mathrm{mV}$. Last, measuring the integral of the calcium channel current for the instant repolarization shows a steady increase in the amount of calcium entering the nerve terminal as the afterpotential becomes more depolarized (Fig. 5E). However, the integral of the calcium current in response to a symmetrical stimulation or a physiological AP-like stimulation is not affected by afterpotentials (Fig. 5E). These results indicate that physiological repolarization rates appear to largely eliminate the effects of afterpotentials on the presynaptic voltage-gated calcium channel response.

\section{Electrical driving force and gating} changes cancel out during simulations As described in the introduction, the lack of effect of afterpotentials on voltagegated calcium channel currents could be due to basic calcium channel gating properties coupled with simultaneous changes in the electrical driving force for calcium entry (Pattillo et al., 1999). To further determine whether these calcium channel properties are sufficient to explain why afterpotentials do not affect calcium channel activity after a ramped repolarization, we simulated the voltage-gated calcium channel activity in response to an AP-like stimulation that ended with an ADP or AHP. Our simulation used a linear sixstate Markovian model (see Materials and Methods and Fig. 6E) consisting of fourclosed states that precede a single open state, with voltage-dependent rate constants governing the transitions between each state (Bischofberger et al., 2002). An additional voltage-dependent nonconducting state was added after the open state to allow accurate simulation of a small residual current that is present in the presynaptic calcium channel responses. The rate constants used for the kinetic transitions (Fig. 6E) were determined by comparing the simulated responses to a recorded calcium channel current in response to an AP-like stimulus in the calyx of Held (Fig. 6A). The onset of the simulated calcium channel responses do not overlap with the recorded response due to a small transient upward component that appears at the beginning of the recorded calcium channel response. The upward component does not appear in the simulated data, which is consistent with the idea that this portion of the response is due to gating currents from calcium channels and other voltage-gated channels in the calyx (Borst and Sakmann, 1998). This upward component appears to create a slight delay in the apparent onset of the calcium channel response. We therefore aligned the start time of simulated and 
A
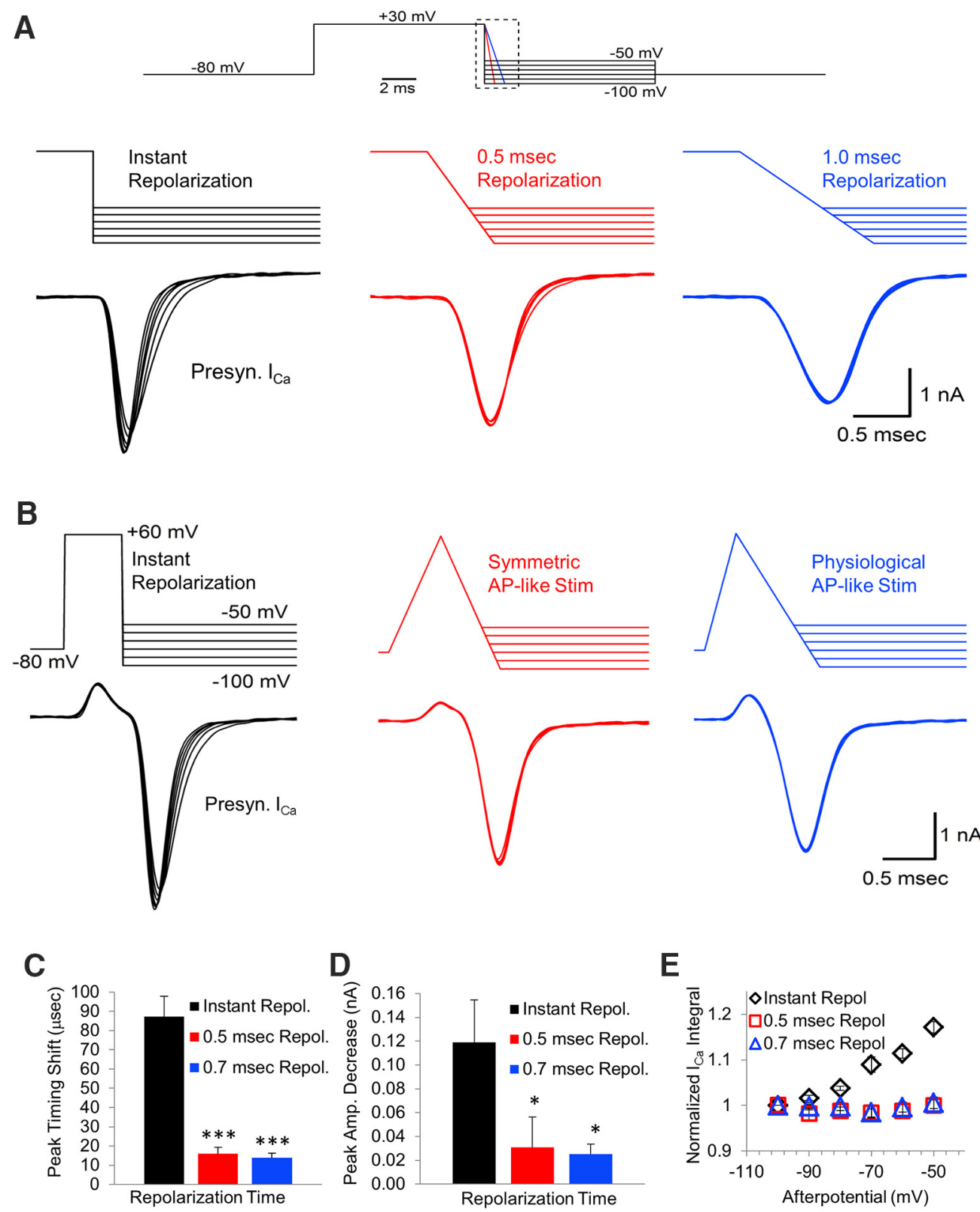

E

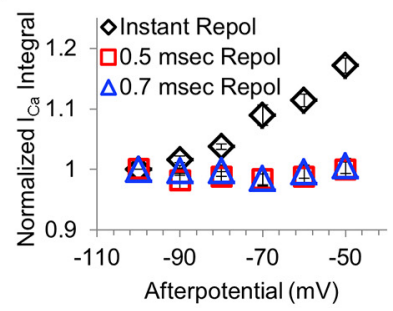

Figure 5. Ramped repolarization acts to reduce or eliminate effects of afterpotentials. $A$, Presynaptic calcium channels were activated by a $10 \mathrm{~ms}$ depolarization to $+30 \mathrm{mV}$ and then repolarized instantly (left trace), over $0.5 \mathrm{~ms}$ (center trace), or over $1.0 \mathrm{~ms}$ (right trace). These repolarizations ended with afterpotentials between -50 and $-100 \mathrm{mV}$ in $10 \mathrm{mV}$ increments. $\boldsymbol{B}$, Timing of the peak of the presynaptic calcium channel response to afterpotentials at -100 and $-50 \mathrm{mV}$ after an instant repolarization versus AP-like stimulation with 0.5 or $0.7 \mathrm{~ms}$ repolarization. $C$, Time from the onset to the peak amplitude of the calcium channel response to afterpotentials at -100 and $-50 \mathrm{mV}$ after an instant repolarization versus 0.5 or $0.7 \mathrm{~ms}$ repolarization. $\boldsymbol{D}$, Peak amplitude of the calcium channel response to afterpotentials at -100 and $-50 \mathrm{mV}$ after an instant repolarization versus 0.5 or $0.7 \mathrm{~ms}$ repolarization. $\boldsymbol{E}$, Normalized integral of the calcium channel response to afterpotentials after an instant repolarization, a $0.5 \mathrm{~ms}$ repolarization, or a $0.7 \mathrm{~ms}$ repolarization.

actual data and focused on the peak timing, peak amplitude, and the decay of the calcium channel response to evaluate the rate constants for the simulation. Using this model, we first tested the effects of afterpotentials on an instant depolarization (Fig. 6B). In agreement with our experimental results, the peak amplitude and the deactivation rate of the simulated calcium channel response steadily decrease as the afterpotential becomes less negative. Next, we simulated the voltage-gated calcium channel current generated by an AP-like stimulus that ended at either $-100 \mathrm{mV}$ or $-60 \mathrm{mV}$ to compare the AHP and ADP responses (Fig. 6C). Similar to our experimental data, the simulation shows little to no effect of afterpotentials on the calcium channel response. The onset of the $-60 \mathrm{mV}$ ADP is marked by an arrow in the figure.
Any effects of afterpotentials on the simulated response would therefore occur in the portion of the trace after the arrow. The lack of effect of afterpotentials on the simulated data indicates that basic mechanisms of calcium channel gating and electrical driving force are sufficient to explain why afterpotentials do not alter the calcium channel response in the presynaptic recordings. However, a complete balance between calcium channel gating and calcium driving force is not a necessary or default outcome based on the simulation. Some rate constant combinations are able to fit the data adequately in the absence of an afterpotential, but do not provide a good fit to the afterpotential portion of the simulation when afterpotential stimuli are tested. This is also evident experimentally in that voltage-gated calcium channels 
A

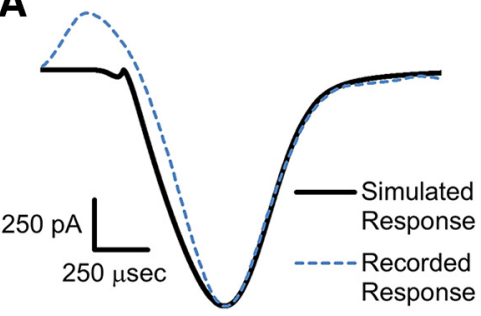

C

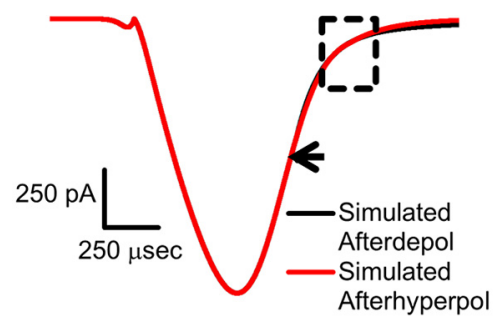

D

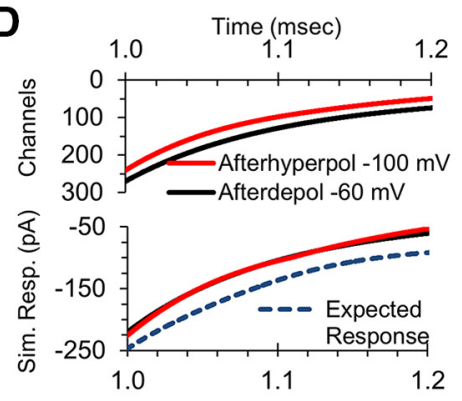

E

\begin{tabular}{|c|c|c|c|c|}
\hline$\alpha_{12}\left(V_{1}\right)$ & $\alpha_{23}\left(V_{2}\right)$ & $\alpha_{34}\left(V_{3}\right)$ & $\alpha_{45}\left(V_{4}\right)$ & $\alpha_{56}\left(V_{5}\right)$ \\
\hline$\alpha_{12}=6 \mathrm{~ms}^{-1}$ & $\alpha_{23}=5 \mathrm{~ms}^{-1}$ & $\alpha_{34}=4 \mathrm{~ms}^{-1}$ & $\alpha_{45}=23 \mathrm{~ms}^{-1}$ & $\alpha_{56}=0.2 \mathrm{~ms}^{-1}$ \\
\hline$\beta_{21}=8 \mathrm{~ms}^{-1}$ & $\beta_{32}=6 \mathrm{~ms}^{-1}$ & $\beta_{43}=9 \mathrm{~ms}^{-1}$ & $\beta_{54}=4.3 \mathrm{~ms}^{-1}$ & $\beta_{65}=0.25 \mathrm{~ms}^{-1}$ \\
\hline$V_{1}=30 \mathrm{mV}$ & $\mathrm{V}_{2}=30 \mathrm{mV}$ & $V_{3}=22 \mathrm{mV}$ & $\mathrm{V}_{4}=60 \mathrm{mV}$ & $\mathrm{V}_{4}=30 \mathrm{mV}$ \\
\hline
\end{tabular}

Figure 6. Simulations of voltage-gated calcium channel response to afterpotentials. $\boldsymbol{A}$, Simulated response to an AP-like stimulation compared with a recorded response to an AP-like stimulation. $\boldsymbol{B}$, Simulated response to an instant repolarization that returns to the baseline level of $-80 \mathrm{mV}$ (blue trace) or with an afterpotential of -60 (black trace) or $-100 \mathrm{mV}$ (red trace). Scale bars, $500 \mathrm{pA}$ and $100 \mu \mathrm{s}$. C, Simulated response to an AP-like stimulation that ended in an ADP of $-60 \mathrm{mV}$ (black trace) or with an AHP of $-100 \mathrm{mV}$. Arrow indicates where the ADP begins in the simulated response. Boxed region is centered at the beginning of the AHP. D, Simulation of the number of calcium channels that are open during the boxed region in $C$ in response to $-60 \mathrm{mV}$ (black trace) or $-100 \mathrm{mV}$ afterpotential (red trace). Bottom shows the simulated calcium channel current during this time. Expected response is the current that would occur for the $-60 \mathrm{mV}$ response in the absence of the reduced electrical driving force. $\boldsymbol{E}$, Gating scheme and the rate constants of the Markovian model used for the simulations are shown (see methods).

have been shown to be affected by ADPs, but not AHPs, in other neurons (Pattillo et al., 1999).

Our simulations are consistent with the idea that changes in the peak amplitude and changes in the deactivation rate both occur across a large range of afterpotentials, but they counteract each other dynamically and result in a calcium channel response that is not altered by afterpotentials. To investigate this, we looked at the number of channels opened by an ADP versus an AHP (Fig. $6 D$, top). These data correspond to a $0.2 \mathrm{~ms}$ portion of the simulated responses that occurs within the dashed-line box in Figure $6 C$. During this time, the ADP stimulus is at $-60 \mathrm{mV}$ and the AHP stimulus transitions from a ramped voltage stimulus to a constant $-100 \mathrm{mV}$ stimulus. The simulation shows that, despite a difference in the number of open channels between the two stimuli, there is no difference in the current they produce (Fig. 6D, bottom, red and black traces). Next, we calculated the expected response based on the number of additional channels that are opened during the ADP (Fig. 6D, bottom, dashed cyan trace). To determine how this would affect the amount of calcium entering the nerve terminal, we averaged 10 calcium channel responses to an AP-like stimulation and scaled up the response based on our simulations. Comparing the area of the expected response to the area of the actual response shows an $\sim 10 \%$ increase in total calcium entry (mean $=10.2 \pm 2.3 \%$; $n=6$ presynaptic recordings, 60 traces total). This is similar to the increase in calcium entry seen for instant repolarizations with a $-60 \mathrm{mV}$ afterpotential compared with the response produced by a $-100 \mathrm{mV}$ afterpotential (Fig. 5E). Therefore, without the compensation between calcium influx and channel gating, the difference in calcium channel activation in response to afterpotentials after an AP-like stimulation would affect calcium entry by an amount that would have a large effect on the release of neurotransmitter and the postsynaptic response.

In summary, the simulations show that the reduced channel activation produced by an AHP can be compensated by the increased calcium driving force. Similarly, the increased calcium channel activity that occurs during an ADP fails to produce a larger calcium current due to the decreased electrical driving force. This shows that a physiological repolarization is capable of causing both a change in driving force and a change in deactivation rate that can effectively cancel each other out over a large range of afterpotentials. This mechanism acts to minimize the direct effects that a change in membrane potential occurring at the end of the repolarization phase of the $\mathrm{AP}$ would have on the presynaptic calcium current and synaptic transmission.

\section{Discussion}

We show here that the membrane potential reached at the end of an AP does not alter the presynaptic calcium current or synaptic transmission if it remains below $-40 \mathrm{mV}$, the approximate activation threshold for voltage-gated calcium channels in the calyx of Held (Schneggenburger and Forsythe, 2006). The altered calcium channel response above $-40 \mathrm{mV}$ appears to occur through the reactivation of voltage-gated calcium channels. This finding was unexpected given that numerous studies have demonstrated that even small changes in the repolarization phase of the presynaptic AP produce changes in calcium entry that can have large effects on neurotransmitter release (Augustine, 1990; Wheeler et al., 1996; Sabatini and Regehr, 1997; Geiger and Jonas, 2000; Lien and Jonas, 2003; Yang and Wang, 2006). Therefore, the repolarization phase of the AP has been associated previously with modulating, not stabilizing, the presynaptic calcium influx and the postsynaptic response.

Although afterpotentials affect the driving force for calcium entry, our results and simulations indicate that this is compensated for by a change in the deactivation rate of calcium channels such that the net influx of calcium is nearly unchanged (Figs. $5 E$, $6 D)$. An earlier study using somatic recordings from chick ciliary ganglia proposed this mechanism to explain results from an AHP experiment (Pattillo et al., 1999). In this work, a $5 \mathrm{mV}$ AHP was found to have only a small effect on the calcium current and the investigators insightfully stated that an increase in the driving force and channel deactivation may be balanced in their contribution to the calcium current. However, they also found that a broadening in the repolarization before the end of the AP-like 
stimulus, which is effectively an ADP, did affect the calcium currents. Here, we demonstrate that this mechanism of balanced gating and driving force can occur in a nerve terminal over a wide range of afterpotentials and it can act to stabilize neurotransmitter release (Figs. $3 A-D, 4 C-E$ ). This mechanism can extend to fairly large ADPs in the nerve terminal (Fig. $2 C$ ) and can prevent changes in the membrane potential from affecting the calcium current (Fig. 2E-F).

Voltage-gated calcium channels are typically studied by applying voltage jumps from the resting potential to determine the voltage sensitivity, current amplitude, and kinetics of calcium channel responses (Hagiwara and Byerly, 1981; Tsien, 1983). Using voltage jumps, afterpotentials have been shown to affect the presynaptic calcium channel tail current (Borst and Sakmann, 1998; Bischofberger et al., 2002). Voltage jumps ending with an AHP show an increase in the tail current amplitude due to the increased electrical driving force, along with a faster deactivation rate due to the voltage sensitivity of channel gating. For the same reasons, an $\mathrm{ADP}$ at the end of an instant voltage jump produces a current with a smaller peak amplitude and slower deactivation rate. Here, we used simultaneous presynaptic and postsynaptic recordings to show that presynaptic voltage jumps ending with an ADP produce a larger amount of neurotransmitter release than voltage steps ending with an AHP. This finding agrees with work from others showing that the calcium current duration and total calcium influx are more important than the peak amplitude of the calcium current in determining the size of the postsynaptic response (Borst and Sakmann, 1999; Bollmann and Sakmann, 2005; Yang and Wang, 2006). This is consistent with the existence of some vesicles that are tightly coupled to calcium channels and a larger population of vesicles that are slightly further from calcium channels at this stage of development (Eggermann et al., 2012). The increased distance between the calcium channels and the vesicles favors release when a larger amount of calcium enters the nerve terminal due to increased duration of calcium channel activity (Wang and Augustine, 2014). In the voltage-jump experiments, the ADP affects gating, which allows a larger number of calcium channels to be open and a larger amount of calcium to enter compared with the response generated by an AHP.

In contrast to the effects shown for instant voltage jumps, afterpotentials after the AP-like stimulation or after actual APs have a negligible effect on calcium channel response and no effect on neurotransmitter release despite the $\sim 40 \mathrm{mV}$ difference between ADPs and AHPs. Therefore, the physiological duration of the AP repolarization plays a major role in stabilizing the calcium channel response and subsequent neurotransmitter release. Our simulations replicate the finding that afterpotentials do not affect the presynaptic calcium channel current, allowing us to conclude that a dynamic balance between gating and driving force can prevent afterpotentials from affecting the calcium current and subsequent neurotransmitter release. In addition, limiting the entry of calcium reduces the need to buffer and eventually extrude intraterminal calcium by ATP-dependent mechanisms (Kim et al., 2005).

Our experiments were done on P8 to P12 animals. By P16, the presynaptic AP shows a twofold decrease in half-width time (Taschenberger et al., 2002). At physiological temperatures, the AP shows a similar decrease in half-width time and the peak amplitude is slightly decreased (Kushmerick et al., 2006). Afterpotentials are present at both physiological temperatures and at later stages of development. Although the AP half-width is decreased at physiological temperatures and at later developmental stages, it is not an instant voltage jump. In our experiments, the stimulus had a repolarization time of $0.7 \mathrm{~ms}$. However, we also tested the effects of afterpotentials on AP-like stimuli with a 0.5 $\mathrm{ms}$ repolarization (Fig. $5 B-E$ ). This indicates that the balanced gating and driving force mechanism should stabilize, or at least contribute to stabilizing, the calcium current at later developmental stages.

It has been shown previously that voltage jumps that begin at $-80 \mathrm{mV}$ or $-60 \mathrm{mV}$ and return to $-80 \mathrm{mV}$ produce matching calcium currents (Awatramani et al., 2005). Together with our finding that afterpotentials do not affect the calcium channel response to an AP-like stimulation, this suggests that a brief change in the resting potential would not affect the presynaptic calcium channel response. In support of this, we show that calcium currents generated by an AP-like stimulus closely match when the resting potential starts and ends at $-100 \mathrm{mV}$ or starts and ends at $-60 \mathrm{mV}$ (Fig. 2E,F). Interestingly, a prolonged change ( $\geq 1 \mathrm{~s}$ ) in resting potential by as little as $10 \mathrm{mV}$ has been shown to generate a low level of calcium channel activity that produces a total summed current of $<1 \mathrm{pA}$ and increases the resting calcium concentration (Awatramani et al., 2005). However, the direct activation of calcium channels by an AP-like stimulation is not affected appreciably by changes in the presynaptic resting potential. This has important implications for synaptic transmission because several different types of currents, including afterpotentials, can produce a brief change the membrane potential of presynaptic terminals. The mechanism of balanced gating and driving force can act to stabilize the calcium currents and neurotransmitter release when changes in the presynaptic membrane potential are below the level that activates the voltagegated calcium channels.

Recordings at physiological temperature also produce a depolarization of the resting potential by $\sim 8 \mathrm{mV}$ (Kim and von Gersdorff, 2012). Although this could have a small effect on the amplitude of the afterpotentials, our results show that afterpotentials between -100 and $-40 \mathrm{mV}$ (Fig. $2 \mathrm{~B}, C$ ) do not affect the calcium current. In addition, our data show that a 40 $\mathrm{mV}$ change in the resting potential before the AP starts does not affect the calcium current generated by an AP-like stimulus (Fig. 2E, F). Therefore, the change in the membrane potential at physiological temperatures should not affect the calcium current.

The calyx of Held nerve terminal contains P/Q-, N-, and R-type voltage-gated calcium channels that are responsible for initiating vesicle fusion and neurotransmitter release (Wu et al., 1999; Sheng et al., 2012). These channels comprise the CaV2 family of voltage-gated calcium channels that are responsible for triggering fast synaptic transmission throughout the brain (Reid et al., 2003). CaV2 calcium channels are present in many nerve terminals and P/Q-type calcium channels are important in triggering neurotransmitter release in many areas of the brain. This indicates that the mechanism that stabilizes calcium channel activity at the end of the AP could apply to other presynaptic terminals in the brain. Unlike typical voltage jumps, our results show that the physiological repolarization of the AP acts to reduce or eliminate the effects that changes in the membrane potential, such as those generated by an afterpotential, would have on calcium entry. Given that $\mathrm{CaV} 2$ channels appear to be responsible for triggering vesicular release in many nerve terminals, we believe that this mechanism of balanced driving force and channel gating should help to stabilize synaptic transmission at other nerve terminals with similar presynaptic calcium channel composition. 


\section{References}

Alle H, Geiger JR (2006) Combined analog and action potential coding in hippocampal mossy fibers. Science 311:1290-1293. CrossRef Medline

Augustine GJ (1990) Regulation of transmitter release at the squid giant synapse by presynaptic delayed rectifier potassium current. J Physiol 431: 343-364. CrossRef Medline

Awatramani GB, Price GD, Trussell LO (2005) Modulation of transmitter release by presynaptic resting potential and background calcium levels. Neuron 48:109-121. CrossRef Medline

Barnes-Davies M, Forsythe ID (1995) Pre- and postsynaptic glutamate receptors at a giant excitatory synapse in rat auditory brainstem slices. J Physiol 488:387-406. CrossRef Medline

Bean BP (2007) The action potential in mammalian central neurons. Nat Rev Neurosci 8:451-465. CrossRef Medline

Bischofberger J, Geiger JR, Jonas P (2002) Timing and efficacy of Ca2+ channel activation in hippocampal mossy fiber boutons. J Neurosci 22: 10593-10602. Medline

Bollmann JH, Sakmann B (2005) Control of synaptic strength and timing by the release-site Ca2+ signal. Nat Neurosci 8:426-434. Medline

Bollmann JH, Sakmann B, Borst JG (2000) Calcium sensitivity of glutamate release in a calyx-type terminal. Science 289:953-957. CrossRef Medline

Borst JG, Sakmann B (1998) Calcium current during a single action potential in a large presynaptic terminal of the rat brainstem. J Physiol 506:143157. CrossRef Medline

Borst JG, Sakmann B (1999) Effect of changes in action potential shape on calcium currents and transmitter release in a calyx-type synapse of the rat auditory brainstem. Philos Trans R Soc Lond B Biol Sci 354:347-355. CrossRef Medline

Borst JG, Helmchen F, Sakmann B (1995) Pre- and postsynaptic whole-cell recordings in the medial nucleus of the trapezoid body of the rat. J Physiol 489:825-840. CrossRef Medline

Chen Z, Das B, Nakamura Y, DiGregorio DA, Young SM Jr (2015) Ca2+ channel to synaptic vesicle distance accounts for the readily releasable pool kinetics at a functionally mature auditory synapse. J Neurosci 35 : 2083-2100. CrossRef Medline

Debanne D (2004) Information processing in the axon. Nat Rev Neurosci 5:304-316. CrossRef Medline

Debanne D, Campanac E, Bialowas A, Carlier E, Alcaraz G (2011) Axon physiology. Physiol Rev 91:555-602. CrossRef Medline

Eggermann E, Bucurenciu I, Goswami SP, Jonas P (2012) Nanodomain coupling between $\mathrm{Ca}(2)(+)$ channels and sensors of exocytosis at fast mammalian synapses. Nat Rev Neurosci 13:7-21. CrossRef Medline

Fernández-Chacón R, Königstorfer A, Gerber SH, García J, Matos MF, Stevens CF, Brose N, Rizo J, Rosenmund C, Südhof TC (2001) Synaptotag$\min$ I functions as a calcium regulator of release probability. Nature 410: 41-49. CrossRef Medline

Forsythe ID, Tsujimoto T, Barnes-Davies M, Cuttle MF, Takahashi T (1998) Inactivation of presynaptic calcium current contributes to synaptic depression at a fast central synapse. Neuron 20:797-807. CrossRef Medline

Gasser HS, Erlanger J (1930) The ending of the axon action potential, and its relation to the other events in nerve activity. Am J Physiol 94:247-277.

Geiger JR, Jonas P (2000) Dynamic control of presynaptic Ca(2+) inflow by fast-inactivating $\mathrm{K}(+)$ channels in hippocampal mossy fiber boutons. Neuron 28:927-939. CrossRef Medline

Hagiwara S, Byerly L (1981) Calcium channel. Annu Rev Neurosci 4:69125. CrossRef Medline

Han Y, Babai N, Kaeser P, Südhof TC, Schneggenburger R (2015) RIM1 and RIM2 redundantly determine $\mathrm{Ca} 2+$ channel density and readily releasable pool size at a large hindbrain synapse. J Neurophysiol 113:255-263. CrossRef Medline

Hoppa MB, Gouzer G, Armbruster M, Ryan TA (2014) Control and plasticity of the presynaptic action potential waveform at small CNS nerve terminals. Neuron 84:778-789. CrossRef Medline

Kim JH, von Gersdorff H (2012) Suppression of spikes during posttetanic hyperpolarization in auditory neurons: the role of temperature, $\mathrm{I}(\mathrm{h})$ currents, and the $\mathrm{Na}(+)-\mathrm{K}(+)$-ATPase pump. J Neurophysiol 108:19241932. CrossRef Medline

Kim JH, Sizov I, Dobretsov M, von Gersdorff H (2007) Presynaptic Ca2+ buffers control the strength of a fast post-tetanic hyperpolarization mediated by the alpha3 $\mathrm{Na}(+) / \mathrm{K}(+)$-ATPase. Nat Neurosci 10:196-205. CrossRef Medline

Kim JH, Kushmerick C, von Gersdorff H (2010) Presynaptic resurgent Na+ currents sculpt the action potential waveform and increase firing reliability at a CNS nerve terminal. J Neurosci 30:15479-15490. CrossRef Medline

Kim MH, Korogod N, Schneggenburger R, Ho WK, Lee SH (2005) Interplay between $\mathrm{Na}+/ \mathrm{Ca} 2+$ exchangers and mitochondria in $\mathrm{Ca} 2+$ clearance at the calyx of Held. J Neurosci 25:6057-6065. CrossRef Medline

Kole MH, Letzkus JJ, Stuart GJ (2007) Axon initial segment Kv1 channels control axonal action potential waveform and synaptic efficacy. Neuron 55:633-647. CrossRef Medline

Kopp-Scheinpflug C, Steinert JR, Forsythe ID (2011) Modulation and control of synaptic transmission across the MNTB. Hear Res 279:22-31. CrossRef Medline

Kushmerick C, Renden R, von Gersdorff H (2006) Physiological temperatures reduce the rate of vesicle pool depletion and short-term depression via an acceleration of vesicle recruitment. J Neurosci 26:1366-1377. CrossRef Medline

Lewis AH, Raman IM (2014) Resurgent current of voltage-gated $\mathrm{Na}(+)$ channels. J Physiol 592:4825-4838. CrossRef Medline

Lien CC, Jonas P (2003) Kv3 potassium conductance is necessary and kinetically optimized for high-frequency action potential generation in hippocampal interneurons. J Neurosci 23:2058-2068. Medline

Lin KH, Oleskevich S, Taschenberger H (2011) Presynaptic Ca2+ influx and vesicle exocytosis at the mouse endbulb of Held: a comparison of two auditory nerve terminals. J Physiol 589:4301-4320. CrossRef Medline

Lin KH, Erazo-Fischer E, Taschenberger H (2012) Similar intracellular $\mathrm{Ca} 2+$ requirements for inactivation and facilitation of voltage-gated $\mathrm{Ca} 2+$ channels in a glutamatergic mammalian nerve terminal. J Neurosci 32:1261-1272. CrossRef Medline

MacDermott AB, Role LW, Siegelbaum SA (1999) Presynaptic ionotropic receptors and the control of transmitter release. Annu Rev Neurosci 22: 443-485. CrossRef Medline

Meir A, Ginsburg S, Butkevich A, Kachalsky SG, Kaiserman I, Ahdut R, Demirgoren S, Rahamimoff R (1999) Ion channels in presynaptic nerve terminals and control of transmitter release. Physiol Rev 79:1019-1088. Medline

Paradiso K, Wu LG (2009) Small voltage changes at nerve terminals travel up axons to affect action potential initiation. Nat Neurosci 12:541-543. CrossRef Medline

Pattillo JM, Artim DE, Simples JE Jr, Meriney SD (1999) Variations in onset of action potential broadening: effects on calcium current studied in chick ciliary ganglion neurones. J Physiol 514:719-728. CrossRef Medline

Reid CA, Bekkers JM, Clements JD (2003) Presynaptic Ca2+ channels: a functional patchwork. Trends Neurosci 26:683-687. CrossRef Medline

Rudy B, McBain CJ (2001) Kv3 channels: voltage-gated K+ channels designed for high-frequency repetitive firing. Trends Neurosci 24:517-526. CrossRef Medline

Sabatini BL, Regehr WG (1997) Control of neurotransmitter release by presynaptic waveform at the granule cell to Purkinje cell synapse. J Neurosci 17:3425-3435. Medline

Schneggenburger R, Forsythe ID (2006) The calyx of Held. Cell Tissue Res 326:311-337. CrossRef Medline

Schneggenburger R, Neher E (2000) Intracellular calcium dependence of transmitter release rates at a fast central synapse. Nature 406:889-893. CrossRef Medline

Sheng J, He L, Zheng H, Xue L, Luo F, Shin W, Sun T, Kuner T, Yue DT, Wu LG (2012) Calcium-channel number critically influences synaptic strength and plasticity at the active zone. Nat Neurosci 15:998-1006. CrossRef Medline

Shu Y, Hasenstaub A, Duque A, Yu Y, McCormick DA (2006) Modulation of intracortical synaptic potentials by presynaptic somatic membrane potential. Nature 441:761-765. CrossRef Medline

Stanley EF, Goping G (1991) Characterization of a calcium current in a vertebrate cholinergic presynaptic nerve terminal. J Neurosci 11:985-993. Medline

Taschenberger H, Leão RM, Rowland KC, Spirou GA, von Gersdorff H (2002) Optimizing synaptic architecture and efficiency for highfrequency transmission. Neuron 36:1127-1143. CrossRef Medline

Tsien RW (1983) Calcium channels in excitable cell membranes. Annu Rev Physiol 45:341-358. CrossRef Medline 
Turecek R, Trussell LO (2001) Presynaptic glycine receptors enhance transmitter release at a mammalian central synapse. Nature 411:587-590. CrossRef Medline

von Gersdorff H, Borst JG (2002) Short-term plasticity at the calyx of Held. Nat Rev Neurosci 3:53-64. Medline

Wang LY, Augustine GJ (2014) Presynaptic nanodomains: a tale of two synapses. Front Cell Neurosci 8:455. CrossRef Medline

Wheeler DB, Randall A, Tsien RW (1996) Changes in action potential duration alter reliance of excitatory synaptic transmission on multiple types of Ca2+ channels in rat hippocampus. J Neurosci 16:22262237. Medline

Wu LG, Westenbroek RE, Borst JG, Catterall WA, Sakmann B (1999) Calcium channel types with distinct presynaptic localization couple differen- tially to transmitter release in single calyx-type synapses. J Neurosci 19: 726-736. Medline

Wu XS, Sun JY, Evers AS, Crowder M, Wu LG (2004) Isoflurane inhibits transmitter release and the presynaptic action potential. Anesthesiology 100:663-670. CrossRef Medline

Yang YM, Wang LY (2006) Amplitude and kinetics of action potentialevoked $\mathrm{Ca} 2+$ current and its efficacy in triggering transmitter release at the developing calyx of Held synapse. J Neurosci 26:5698-5708. CrossRef Medline

Yang YM, Wang W, Fedchyshyn MJ, Zhou Z, Ding J, Wang LY (2014) Enhancing the fidelity of neurotransmission by activity-dependent facilitation of presynaptic potassium currents. Nat Commun 5:4564. CrossRef Medline 